\title{
Accurate prediction of protein secondary structure and solvent accessibility by consensus combiners of sequence and structure information
}

\author{
Gianluca Pollastri*, Alberto JM Martin, Catherine Mooney and
} Alessandro Vullo

Address: Complex and Adaptive Systems Laboratory, School of Computer Science and Informatics, University College Dublin, Belfield, Dublin 4, Ireland

Email: Gianluca Pollastri* - gianluca.pollastri@ucd.ie; Alberto JM Martin - albertoj@ucd.ie; Catherine Mooney - catherine.mooney@ucd.ie; Alessandro Vullo - alessandro.vullo@ucd.ie

* Corresponding author

Published: 14 June 2007

BMC Bioinformatics 2007, 8:20I doi:I0.1 186/I47I-2105-8-20I

This article is available from: http://www.biomedcentral.com/I47I-2105/8/20।

(c) 2007 Pollastri et al; licensee BioMed Central Ltd.

This is an Open Access article distributed under the terms of the Creative Commons Attribution License (http://creativecommons.org/licenses/by/2.0), which permits unrestricted use, distribution, and reproduction in any medium, provided the original work is properly cited.

\begin{abstract}
Background: Structural properties of proteins such as secondary structure and solvent accessibility contribute to three-dimensional structure prediction, not only in the $a b$ initio case but also when homology information to known structures is available. Structural properties are also routinely used in protein analysis even when homology is available, largely because homology modelling is lower throughput than, say, secondary structure prediction. Nonetheless, predictors of secondary structure and solvent accessibility are virtually always $a b$ initio.
\end{abstract}

Results: Here we develop high-throughput machine learning systems for the prediction of protein secondary structure and solvent accessibility that exploit homology to proteins of known structure, where available, in the form of simple structural frequency profiles extracted from sets of PDB templates. We compare these systems to their state-of-the-art $a b$ initio counterparts, and with a number of baselines in which secondary structures and solvent accessibilities are extracted directly from the templates. We show that structural information from templates greatly improves secondary structure and solvent accessibility prediction quality, and that, on average, the systems significantly enrich the information contained in the templates. For sequence similarity exceeding $30 \%$, secondary structure prediction quality is approximately $90 \%$, close to its theoretical maximum, and 2-class solvent accessibility roughly $85 \%$. Gains are robust with respect to template selection noise, and significant for marginal sequence similarity and for short alignments, supporting the claim that these improved predictions may prove beneficial beyond the case in which clear homology is available.

Conclusion: The predictive system are publicly available at the address http://distill.ucd.ie.

\section{Background}

Protein secondary structure and solvent accessibility predictions are an important stage towards the prediction of protein structure and function. Accurate secondary structure and solvent accessibility information is not only at the core of most $a b$ initio methods for the prediction of 
protein structure (e.g. see [2]) but is also effective in improving the sensitivity of fold recognition methods (e.g. [3-5]), and is routinely used in protein analysis and annotation [6].

Virtually all modern methods for the prediction of protein one-dimensional structural features (i.e. those features which may be represented as a string of the same length as the primary sequence, such as secondary structure and solvent accessibility) are based on machine learning techniques [7-22], and exploit evolutionary information in the form of amino acid frequency profiles extracted from alignments of multiple sequences, generally of unknown structure. The progress of these methods over the last 10 years has been slow, but steady, and is due to numerous factors: the ever-increasing size of training sets; more sensitive methods for the detection of homologues, such as PSI-BLAST [23]; the use of ensembles of multiple predictors trained independently, sometimes tens of them [12]; more sophisticated machine learning techniques (e.g. [14]); a combination of a number of the above [19].

Predictors of secondary structure and solvent accessibility are virtually always $a b$ initio (with very few exceptions, e.g., recently, [22]), meaning that they do not rely directly on similarity to proteins of known structure. In fact, often, much care is taken to try to exclude any detectable similarity between training and test set instances when gauging predictive performances of structural feature predictors. The main reason for this seems to be a short-circuit, which happened early on in the field and was never disputed, between the idea of hypothesis validation by strict training and test set separation (borrowed from statistical learning), and the concept of $a b$ initio prediction. For training and test sets to be strictly distinct, they are required to not only contain different examples (which is all the statistical learning principle dictates, together with independence and identical distribution), but to contain examples that do not show significant sequence identity to one another, as detected by a standard BLAST [23] search. A hint of the historical, more than scientific, nature of this issue is the fact that when subtler algorithms for sequence similarity detection became available (e.g. PSI-BLAST [23]), the criteria for training vs. test set separation did not always change.

Currently over half of all known protein sequences show some detectable degree of similarity to one or more sequences of known structure. Nearly 3/4 of newly deposited structures in the PDB [24] show significant similarity to previously deposited structures [22]. Over $60 \%$ of the queries received by the server Porter [19] in the first six months of year 2006 have potential homologues in the PDB at the moment of submission (PSI-BLAST e-value smaller than 0.01 ), and another $25 \%$ have marginal sim- ilarity to some sequence in the PDB (PSI-BLAST e-value between 0.01 and 10). For the case of clear homology, direct structural information from the homologous proteins can be exploited for the prediction of structural features. For instance, secondary structure extracted from full three-dimensional comparative models is known to be significantly more reliable than secondary structure obtained from $a b$ initio predictors [8,22]. Moreover, even where alignments to PDB structures are of dubious reliability, or too short to reliably imply homology, these may carry information. One of the main sources of improvement for fold recognition and $a b$ initio structure prediction methods over the last few CASP competitions [25-28] has been the reliance on sets of possible conformations for short fragments of chain [28], extracted from the PDB.

There is a number of reasons why direct, machine learning-based predictions of secondary structure or other structural features incorporating homology information are useful: nearly all the most reliable public predictors $[6,9,14,29,30]$ ([22] is an exception, potentially equally reliable, although currently not tested by independent assessors such as EVA [31]) do not take structural information directly into account, which implies that over half of the responses provided to users could be improved, often dramatically; machine learning methods are robust with respect to noise - selecting a template from a set of candidate structures from the PDB may be less of a problem than in traditional comparative modelling, since a set or a profile of templates (possibly conflicting) may be provided to the method, rather than a single template which might be erroneous; machine learning methods are significantly faster than full comparative modelling methods large-scale predictions may be generated with relatively modest computational resources, and feed into structurebased functional similarity algorithms, comparative modelling validation and template selection, protein analysis and proteome annotation efforts; low-similarity, shortalignment based predictions may improve on traditional $a b$ initio ones in fold recognition or even novel fold cases.

Here we develop high-throughput systems for the prediction of protein secondary structure and solvent accessibility, which exploit similarity to proteins of known structure, where available, in the form of simple structural frequency profiles from sets of PDB templates. The systems have two stages: one in which a set of templates for a query sequence is generated based on a similarity search of the PDB; one in which this template information, plus the primary sequence, and evolutionary information in the form of multiple alignments is used as input to an ensemble of recursive neural networks to determine a query's secondary structure and solvent accessibility. Although here we use a simple PSI-BLAST-based protocol to find suitable templates (see Methods section), our sys- 
tems are fully modular and may easily accommodate more sophisticated stages with better sensitivity to remote homology (e.g. [5,32]). It is important to stress that, when homology information is available, the systems we design here do not simply take it as the final answer, but rather use it as a further input. This, on average, leads to significant improvements over extracting secondary structure and solvent accessibility directly from the best single PDB template, and weighed and unweighed averages of the top 10 templates, or all templates identified, suggesting that the combination of sequence and template information carries more information than templates alone. Not surprisingly, when only very high quality templates are available (PSI-BLAST e-value smaller than roughly $10^{-30}$ ), which are almost guaranteed to be close homologues, the improvements become marginal.

We also compare the predictive systems to to their stateof-the-art ab initio counterparts. We show that similarity information, when available, greatly improves prediction quality. For sequence similarity exceeding $30 \%$, prediction quality is nearly at its theoretical maximum. Gains are significant for low sequence similarity when we design specialised systems for this case, and for alignments shorter than 20 residues, outside traditional comparative modelling territory, supporting the claim that these improved predictions may prove beneficial for fold recognition algorithms.

The predictive systems described in this paper are publicly available at the address [1], as part of a suite of predictors of protein structural features. When the user requests secondary structure (Porter) or solvent accessibility (PaleAle) predictions, homology-based results are automatically selected when suitable templates are available. Up to 20,000 queries a day may be served by the 40 CPU cluster hosting the predictors.

\section{Results and Discussion}

The four systems we describe here are: an ab initio secondary structure predictor (Porter [19]) in three classes; the same, but homology-based (Porter_H); an ab initio predictor of relative solvent accessibility in 4 and 2 classes (PaleAle); the same, but homology-based (PaleAle_H). All systems are trained and tested in rigorous 5-fold crossvalidation on the December 2003 25\% pdb_select list [33] (for details, see Methods section). We use this set to make direct comparisons with Porter's published results, and performances as recorded by EVA [31]. The public versions of the servers will undergo regular trainings to keep them up-to-date with the expansion of the PDB.

Porter classifies correctly $79.0 \%$ of all residues. Porter has currently the highest performance of all predictors tested by independent assessor EVA [31], and is ranked first of all methods by EVA based on the combination of pairwise comparisons of servers on identical sets. Overall, Porter_H classifies correctly $85.7 \%$ residues, or nearly $7 \%$ above Porter. If we consider only those residues for which PDB template information is available $(270,110$ out of $344,653)$, Porter_H's performance rises to $88.3 \%$, roughly $9 \%$ above Porter. If we further restrict our observation to those residues for which template information is available with a BLAST e-value of 0.01 or smaller $(250,247$ residues), Porter_H's performance rises to $89.3 \% \quad(9.5 \%$ above Porter). It is worth reminding that the theoretical maximum for secondary structure prediction performance is well below $100 \%$ and is bounded by the intrinsic ambiguity of mapping three-dimensional atom coordinates into secondary structure classes. Any two automated programmes for secondary structure assignment (e.g. $[34,35])$ differ on at least $5 \%$ residues, with up to $20 \%$ residues assigned to different states in some cases [36]. Nonetheless, these larger margins are likely mainly due to different definitions of secondary structures by different automated assignment programs, and only by a smaller amount to actual uncertainties as to what the structure might be. Once a semantics is chosen (e.g., as in our case, DSSP) it is possible to classify secondary structure with an accuracy of more than 90\% [37]. Hence the theoretical maximum for classification accuracy (i.e. classifying as well as an algorithm which takes as input the experimental structure) is likely somewhere in the $90-95 \%$ region. As shown in figure 1, about $90 \%$ residues are correctly classified by Porter_H in the $50 \%-100 \%$ similarity (percent identity) region. Nearly $87 \%$ are correctly classified between 30 and 50\% similarity. Even in the $20-30 \%$ similarity region, Porter_H significantly outperforms Porter ( $82 \%$ average classification performance vs. $79 \%$, with standard deviations of $0.3 \%$ ). For similarity below $20 \%$ Porter_H performs slightly worse than Porter. The two main reasons for this are probably: the better specialisation of the latter system, which is trained on 2171 template-less examples, while the former is trained only on about 500 examples with no or very low quality templates; the fact that PSI-BLAST is increasingly inaccurate as sequence similarity becomes lower and noise in the templates eventually dominates over signal.

\section{Low-similarity templates}

To investigate whether a specialised system can yield improvements when only low-similarity templates are available, we re-trained Porter_H twice, with the further constraint that only templates with at most $30 \%$ and $20 \%$ sequence similarity are adopted, i.e. all PDB templates showing more than $30 \%$ (resp. $20 \%$ ) sequence similarity to the query are eliminated. We refer to the system with maximum 30\% similarity templates as Porter_H30 and with maximum $20 \%$ similarity templates as Porter_H20. The constraint imposed on template quality implies that 


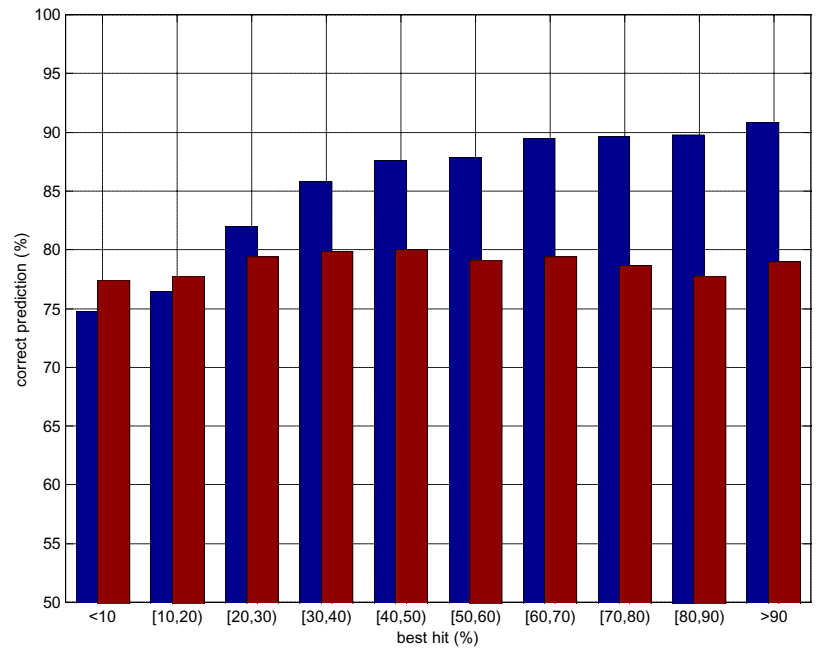

\section{Figure I}

Distribution of secondary structure prediction accuracy as a function of sequence similarity to the best hit in PSI-BLAST templates. The blue bars represent predictions using templates (maximal sequence similarity allowed is $95 \%$ ), the red bars template-less predictions (Porter). See text for details.

many more examples are provided for which no template or only marginal templates are available. Porter_H30's performances in the $0-30 \%$ similarity range increase and improve over Porter for all template lengths (see figure 2). Porter_H30 improves consistently over Porter for sequence similarity greater than $17 \%$. Porter_H2O improves consistently over Porter for sequence similarity greater than $13 \%$. Overall, Porter_H30 is not statistically distinguishable from Porter in the $10-20 \%$ similarity range, while Porter_H20 actually outperforms Porter in this range. These results suggest that, although noisy, PSIBLAST-based templates in the $10-20 \%$ similarity region still retain information that can be sifted out by a machine learning system, provided that enough examples are available. This also suggests that more subtle similarity tools (e.g. [5,32]) are likely to yield better results in marginal/ fold recognition regions if coupled with our predictor. We are in the process of investigating this further point. An example of prediction by Porter_H is reported in Figure 3.

\section{Porter_H vs. templates}

Table 1 reports the comparison between Porter_H and a baseline which simply assigns a residue's secondary structure by copying it from the best template available in the PDB (i.e. that ranked highest by PSI-BLAST). The results are measured only on those residues for which templates exist. Porter_H outperforms the baseline by significant margins for all template qualities allowed: by $2.1 \%$ when trained on templates with sequence similarity of up to $95 \%$; by $6.1 \%$ for similarity of up to $30 \%$; by $9.3 \%$ for similarity of up to $20 \%$.

We also tested different baselines in which, instead of just the top template, respectively, the top 10 templates (as ranked by PSI-BLAST) and all the templates are used to predict the secondary structure of a protein. In both cases the prediction is obtained as a majority vote among the templates covering each residue. We tested both an unweighed vote (i.e. one in which each template counts the same) and a vote in which each template is weighed by its sequence similarity to the query, cubed. The latter weighing scheme is identical to the one used to present the templates to the network (see Methods section for details), and we refer to it as baseline_input. In all cases the predictions are worse than those obtained by only considering the top template (by at least $2 \%$ for the $95 \%$ maximum similarity case, and at least $3 \%$ for the $30 \%$ and $20 \%$ maximum similarity cases), hence at least $4 \%$ worse than Porter_H.

When we consider templates with sequence similarity of up to $95 \%$ and exclude marginal hits (BLAST e-value greater than 0.01), Porter_H still outperforms the baseline by a significant margin, although reduced $(0.8 \%)$. This continues to be true when the threshold for excluding hits is lowered, down to $10^{-40}$, beyond which the differences between the best baseline and the predictors become negligible. Table 4 reports the level of disagreement between

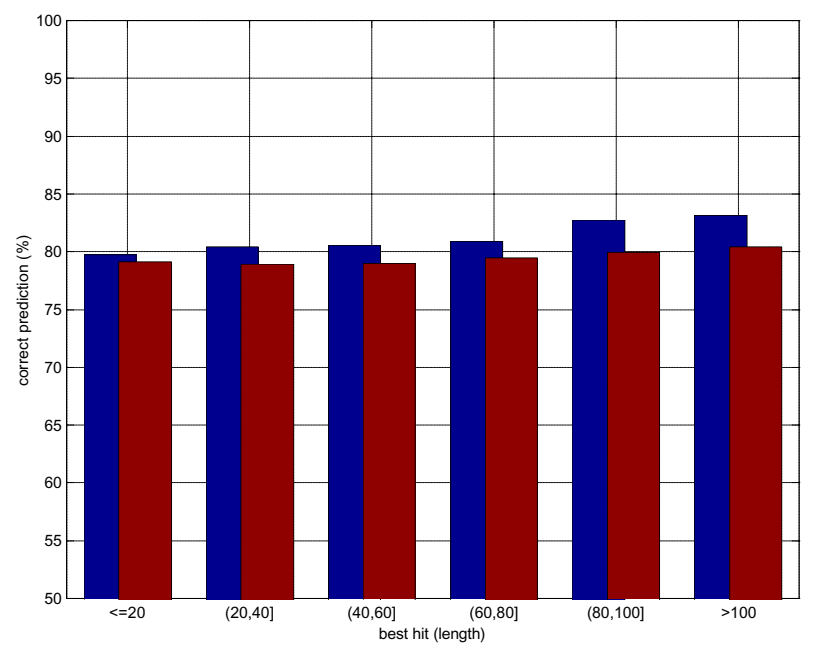

Figure 2

Distribution of secondary structure prediction accuracy as a function of the length of the best hit in PSI-BLAST templates. Maximal $30 \%$ identity between template and query allowed. The blue bars represent predictions using templates, the red bars template-less predictions. See text for details. 


\begin{abstract}
$1 Y L Q A$ CCHHHHHHHHHHHHCCCCEEEEECHHHHCCCCCCCCCEEEEEECHHHHCHHHHHHHHHHHHHHCCCCCEEEEEECHHHHHHHHCCCCCCEEC dSSP CCCCEEEEEEECCCCCCCEEEEECEEEECCCCCCCCCCEEEEEECCCCCCCHHCCEEEEEEEEECCCCEEEEEECHHHHHHHHHHHHHHHCC porter CCCHHHHHHHEECCCCCCEEEEEEEEEECCEECCCCCEEEEEEECCCCCCCCCCCEEEEEEEEECCCCCEEEEECCCHHHHHHHHHHHHHCC porter $h$ CCCHHHHHHHHHHHHCCCCEEEEEECCCCCEEECCSHHHHHHCCHHHHCCCCCCCCCEEEEEC $\ldots \ldots \ldots \ldots \ldots \ldots \ldots \ldots \ldots \ldots$
\end{abstract}

\title{
Figure 3
}

An example of prediction by Porter_H compared to Porter, DSSP assignments, and best template. Best template sequence similarity is $22 \%$. Porter_H correctly identifies the first helix (from the template - strand in Porter), but does not follow the template and assigns correctly the second strand (helix in the template).

Porter_H and baseline_input, which is the baseline it agrees most with. Disagreement is measured simply as the percentage of residues on which Porter_H's and baseline_input's predictions are different. The overall disagreement is $9.9 \%$, which decreases to $8.6 \%$ on residues for which templates exist with $e<0.01$, and grows to over $30 \%$ when templates with $e<0.01$ are excluded.

These results suggest that combining sequence and structure information is a better choice than only relying on templates, i.e. the sequence contains enough information to resolve at least some of the ambiguity contained in sets of templates retrieved by sequence similarity.

The distribution of prediction performances as a function of the quality of the best hit (measured as X-ray resolution + R-factor/20) is shown in figure 4. Homology-based predictions are better for all intervals of quality, but not surprisingly the gains decrease with the decrease in quality of the templates. Somewhat surprisingly in the case of NMR templates the gains (but not the overall prediction performances, possibly due to the different distribution of NMR proteins) are comparable to those obtained with high quality templates from X-ray crystallography.

We also checked whether the presence of membrane proteins in the sets we use for training and testing has any influence on the results. In total there are 64 membrane proteins out of 2171 in the set, covering roughly $5 \%$ of all amino acids. On these proteins Porter_H outperforms Porter by $5.1 \%$ ( $82.1 \%$ correct prediction vs. $77 \%$ ), less than on the whole set. Removing membrane proteins from the set changes the performances of both Porter and
Porter_H by less than $0.1 \%$, and keeps the difference between the methods statistically unchanged.

Lastly, we tested Porter_H on the EVA common2 set as available in November 2004, containing 134 proteins. On this set, a version of Porter retrained from scratch, after having excluded from its training set all sequences with more than $25 \%$ similarity to any sequence in the set, achieves $76.8 \%$ correct prediction, better by at least $1.9 \%$ than all the other servers evaluated. On the same set, a similarly retrained Porter_H achieves $81.5 \%$ correct prediction when templates with more than 95\% sequence similarity to the query are ignored. In this set for 68 out of 134 sequences the best template is below $30 \%$ sequence similarity and for 44 below 20\%.

\section{Solvent Accessibility prediction}

Similar results are obtained for solvent accessibility prediction, as shown in figures 5 and 6 , and table 2. The figures and table refer to 4-class solvent accessibility prediction. The template-less predictor (PaleAle) achieves $53.3 \%$ correct 4 -class prediction. If the 4 classes are reassigned into 2 with a $25 \%$ accessibility threshold (simply by merging the first two classes and the last two classes) the performance rises to $78.9 \%$. Although comparisons on different sets are always not entirely fair, this is at least as good as the most recent 2-class predictors adopting the same class threshold, e.g. [21] (78.1\%), [20] (77.7\%), [38] (78.5\%), [18] (76.7\%).

Table 3 reports a comparison of PaleAle on the Manesh set [39], containing 215 proteins, against a number of other predictors (data from [21]). For this test we retrained

Table I: Performances of the template-based secondary structure predictor (Porter_H) compared with a baseline predictor which copies the secondary structure of the best template (baseline) and with the ab initio secondary structure predictor (Porter).

\begin{tabular}{|c|c|c|c|}
\hline $\max I D$ & baseline & Porter_H & Porter \\
\hline $95 \%$ & $86.2 \%$ & $88.3 \%$ & $79.6 \%$ \\
\hline $30 \%$ & $77.6 \%$ & $83.7 \%$ & $80.2 \%$ \\
\hline $20 \%$ & $73.1 \%$ & $82.4 \%$ & $80.5 \%$ \\
\hline
\end{tabular}

Templates up to $95 \%, 30 \%$ and $20 \%$ maximum similarity allowed (maxID) for baseline and Porter_H. Performances measured only on residues for which a template has been identified. Results in 5-fold cross-validation on the S2I7I set (see text for details). 
PaleAle after having excluded from its training set all sequences with over $25 \%$ sequence similarity to any sequence in the Manesh set (this reduced the size of the set from 2171 to 1662 proteins). After retraining, PaleAle achieves $79.2 \%$ correct prediction on the set $(52.5 \%$ in 4 classes), or at least $1.1 \%$ better than any of the other predictors tested. The template-based predictor (PaleAle_H) classifies correctly $61.8 \%$ of all residues $(82.6 \%$ for the $2-$ class problem), gaining significantly for template identity above 20\%, and slightly underperforming compared to PaleAle for best template identity below 20\%. On the 270,110 residues for which template information is available, the performance grows to $64.8 \%$. On the Manesh set, the 2-class, retrained version of PaleAle_H classifies correctly $86.0 \%$ of all residues $(68.0 \%$ for 4 classes $)$, or $6.8 \%$ better than PaleAle (15.5\% for 4 classes). Gains and losses are all of the same sign and of similar magnitude as in the secondary structure case, counter to the assumption that solvent accessibility is less clearly conserved than secondary structure, hence harder to fathom from templates. Even in the case of solvent accessibility, gains over a baseline predictor copying the accessibility of the best template are large for all levels of maximal sequence similarity allowed: $3.7 \%$ for maximal similarity of $95 \% ; 7.9 \%$ for $30 \% ; 9.9 \%$ for $20 \%$. In all three cases the template-based predictor outperforms, on average, its ab initio variant. For solvent accessibility we also tested different baselines in which the top 10 templates and all templates found by PSI-BLAST are considered, both unweighed and weighed by the cube of their sequence similarity to the query (baseline_input), as in the case of secondary structure. In this case, though, instead of a having a majority vote, for each residue we computed the (weighed or unweighed) average of the solvent accessibility values from the templates and then used this average to determine the solvent accessibility class. As in the secondary structure case, these baselines yielded lower performances than the one based on the top template, by at least $1 \%$ for the $95 \%$ maximum similarity case, and $2 \%$ for both the $30 \%$ and $20 \%$ maximum similarity case, or always at least $3 \%$ worse than PaleAle_H. When we focus only on more reliable templates (PSI-BLAST e-value smaller than 0.01) PaleAle_H still outperforms all the baselines by significant margins (at least 1.2\%) on the residues covered, and disagrees on $14.4 \%$ of residues with its closest baseline (baseline_input. See Table 4). When we tighten the evalue threshold to exclude hits from 0.01 down to $e<10$ 20, PaleAle_H still significantly outperforms all the baselines. Beyond e $<10-20$ PaleAle_H's improvements over the baselines become marginal. As in the case of secondary structure, removing membrane proteins from the sets leaves the results statistically unchanged.

\section{Conclusion}

We have developed high-throughput systems for the prediction of protein secondary structure and solvent accessibility, exploiting similarity to proteins of known structure. These systems, based on machine learning techniques, greatly outperform their $a b$ initio counterparts when PDB templates are available, are capable of combining sequence information and structural information from multiple templates, and outperform simpler strategies such as the extraction of the structural properties in question from the best available template in the PDB, or from weighed and unweighed profiles of templates. Moreover, they are entirely automated, and can be run on multigenomic or bioengineering scales. On a small cluster of machines, hundreds of thousands of protein structural features may be predicted in days.

What is especially encouraging is that performance gains are significant even for marginal sequence similarity when we design specialised systems for this case. This suggests that our strategy may feed into fold recognition systems, which currently rely on $a b$ initio secondary structure predictors. A closed-loop strategy in which the results of fold recognition searches are fed back into the predictors is also possible, and is the object of our current investigation.

All predictive systems are available at the address [1]. Template-based predictions are automatically returned by the secondary structure prediction server (Porter) and the solvent accessibility server (PaleAle) when templates showing more than $20 \%$ sequence similarity to the query are detected. Given the current distribution of queries, this will yield greatly improved predictions for well over half of all requests.

\section{Methods \\ Training set}

The data set used in our simulations is extracted from the December 2003 25\% pdb_select list [33]. We assign each residue's secondary structure and solvent accessibility using the DSSP program [34]. The relative solvent accessibility of a residue is defined as the accessibility in $\AA^{2}$ as computed by DSSP, divided by the maximum observed accessibility for that type of residue. Secondary structure is mapped from the 8 DSSP classes into three classes as follows: H, G, I $\rightarrow$ Helix; E, B $\rightarrow$ Strand; S, T, . $\rightarrow$ Coil. Relative solvent accessibility is mapped into 4 classes where class thresholds are chosen to be maximally informative, i.e. to split the set into (roughly) equally numerous classes: [0\%, $4 \%),[4 \%, 25 \%),[25 \%, 50 \%)$ and $[50 \%, \infty)$ exposed.

We remove all sequences for which DSSP does not produce an output due, for instance, to missing entries (e.g. if only the $\mathrm{C}_{\alpha}$ trace is present in the PDB file) or format 


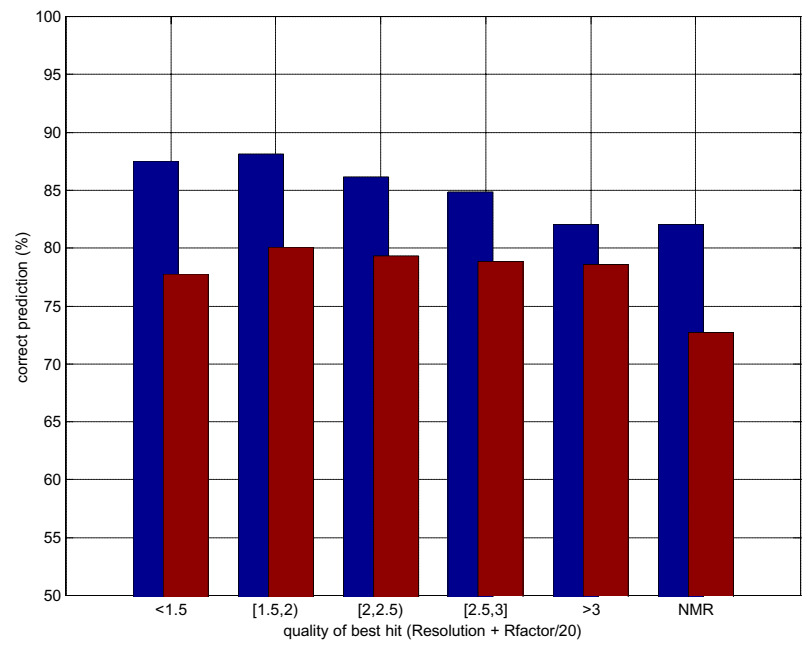

Figure 4

Distribution of secondary structure prediction accuracy as a function of quality of the best hit in PSI-BLAST templates. Quality measured as Resolution+Rfactor/20. The blue bars represent predictions using templates, the red bars templateless predictions (Porter). See text for details.

errors. After processing by DSSP, this set (S2171) contains 2171 proteins and 344,653 amino acids. All the tests reported in this paper are run in 5 -fold cross validation on S2171. The 5 folds are of roughly equal sizes, composed of 434-435 proteins and ranging between 67,345 and 70,098 residues. The datasets are available upon request.

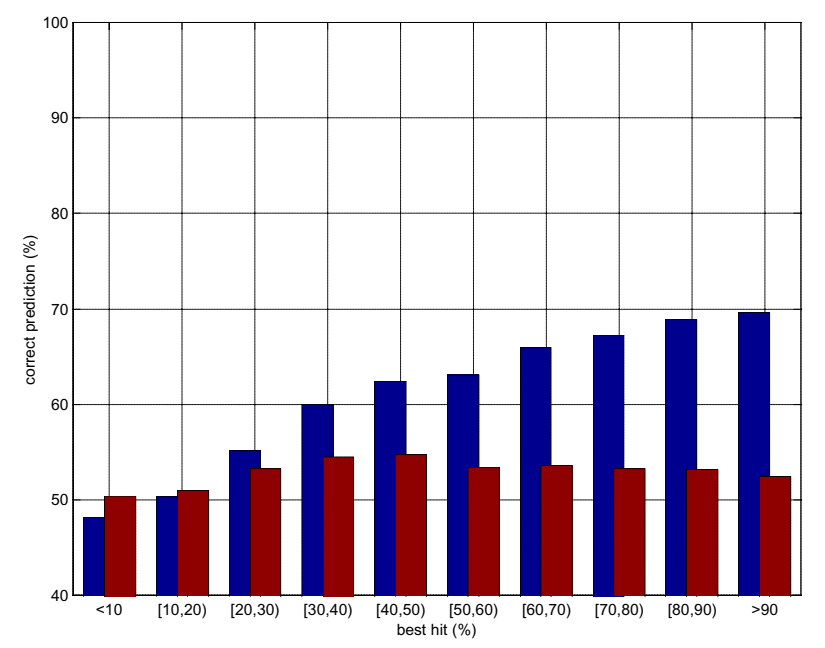

Figure 5

Distribution of 4 -class (4\%, $25 \%$ and $50 \%$ exposed thresholds) solvent accessibility prediction accuracy as a function of sequence similarity to the best hit in PSI-BLAST templates. The blue bars represent predictions using templates (maximal sequence similarity allowed is $95 \%$ ), the red bars template-less predictions. See text for details.
Prediction from a multiple alignment of protein sequences rather than a single sequence has long been recognised as a way to improve prediction accuracy for virtually all protein structural features: secondary structure $[9,10,14,19,22,29,40]$, solvent accessibility $[11,13,15$ $18,20,21]$, beta-sheet pairing $[41,42]$, contact maps [43$45]$, etc. We exploit evolutionary information in the form of frequency profiles compiled from alignments of multiple homologous sequences, extracted from the NR database. Multiple sequence alignments for the S2171 set are extracted from the NR database as available on March 3 2004 containing over 1.4 million sequences. The database is first redundancy reduced at a $98 \%$ threshold, leading to a final 1.05 million sequences. The alignments are generated by three runs of PSI-BLAST [23] with parameters $b=$ 3000 (maximum number of hits),$e=10^{-3}$ (expectation of a random hit) and $h=10^{-10}$ (expectation of a random hit for sequences used to generate the PSSM).

Data sets, training/test folds and multiple alignments are identical to those used to train and test the ab initio secondary structure predictor Porter [19].

\section{Template generation}

For each of the proteins in S2171 we search for structural templates in the PDB. We base our search on PDBFINDERII [46] as available on August 22 2005. An obvious problem arising is that all proteins in the S2171 set are expected to be in PDB (barring name changes), hence every protein will have a perfect template. To avoid this, we exclude from PDBFINDERII every protein that appears in S2171. We also exclude all entries shorter than 10 residues, leading to a final 66,350 chains. Because of the PDBFINDERII origin, only one chain is present in this set for NMR entries.

To generate the actual templates for a protein, we run two rounds of PSI-BLAST against the version of the redundancy-reduced NR database described above, with parameters $b=3000, e=10^{-3}$ and $h=10^{-10}$. We then run a third round of PSI-BLAST against the PDB using the PSSM generated in the first two rounds. In this third round we deliberately use a high expectation parameter $(e=10)$ to include hits that are beyond the usual Comparative Modelling scope $(e<0.01$, at the CASP6 competition [28]). We further remove from each set of hits thus found all those with sequence similarity exceeding $95 \%$ over the whole query, to exclude PDB resubmissions of the same structure at different resolution, other chains in N-mers and close homologues.

The distribution of sequence similarity of the best template, and average template similarity is plotted in figure 7. Roughly $15 \%$ of the proteins have no hits at more than $10 \%$ sequence similarity. About $20 \%$ of all proteins have 


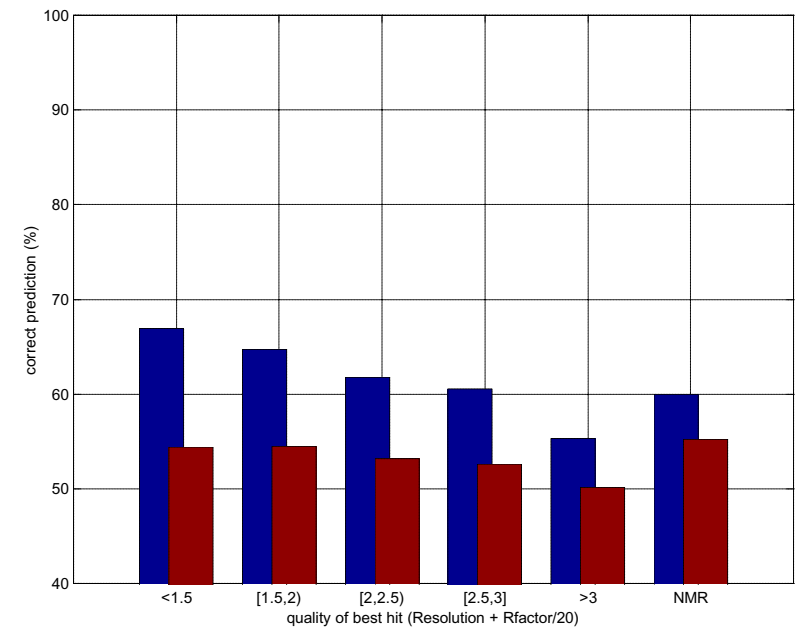

Figure 6

Distribution of 4 -class (4\%, $25 \%$ and $50 \%$ exposed thresholds) solvent accessibility prediction accuracy as a function of quality of the best hit in PSI-BLAST templates. Quality measured as Resolution+Rfactor/20. The blue bars represent predictions using templates, the red bars template-less predictions. See text for details.

at least one very high quality (better than $90 \%$ similarity) entry in their template set.

Although the distribution is not uniform, all similarity intervals are adequately represented: for about $40 \%$ of the proteins no hit is above $30 \%$ similarity; for nearly $20 \%$ of the proteins the best hit is in the $30-50 \%$ similarity interval. Overall 74,543 residues $(21.6 \%$ of the set) are not covered by any template. The average similarity for all PDB hits for each protein, not surprisingly, is generally low: for roughly $75 \%$ of all proteins in S2171 the average identity is below $30 \%$.

To test template-based predictions in marginal similarity conditions we also extract two further template sets from which all hits are excluded that exceed, respectively, 30\% and $20 \%$ sequence similarity. In this case the number of residues not covered by any template climbs, respectively, to 148,124 (43\% of the total) and 193,921 (56.3\%).

\section{Predictive architectures}

To learn the mapping between our input space $\mathcal{I}$ and output space $O$ we use two-layered architectures composed of Bidirectional Recurrent Neural Networks (BRNN)(Also known as 1D-RNN, e.g. in [44]) [10] of the same length $N$ as the amino acid sequence. Similarly to [19] we use BRNNs with shortcut connections. In these BRNNs, connections along the forward and backward hidden chains span more than 1-residue intervals, creating shorter paths between inputs and outputs. These networks take the form:

$$
\begin{gathered}
o_{j}=\mathcal{N}^{(O)}\left(i_{j}, h_{j}^{(F)}, h_{j}^{(B)}\right) \\
h_{j}^{(F)}=\mathcal{N}^{(F)}\left(i_{j}, h_{j-1}^{(F)}, \ldots, h_{j-S}^{(F)}\right) \\
h_{j}^{(B)}=\mathcal{N}^{(B)}\left(i_{j}, h_{j+1}^{(B)}, \ldots, h_{j+S}^{(B)}\right) \\
j=1, \ldots, N
\end{gathered}
$$

where $i_{j}$ (resp. $o_{j}$ ) is the input (resp. output) of the network in position $j$, and $h_{j}^{(F)}$ and $h_{j}^{(B)}$ are forward and backward chains of hidden vectors with $h_{0}^{(F)}=h_{N+1}^{(B)}=0$. We parametrise the output update, forward update and backward update functions (respectively $\mathcal{N}(O), \quad \mathcal{N}(F)$ and $\mathcal{N}(B)$ ) using three two-layered feed-forward neural networks.

\section{Encoding sequence and template information}

Input $i_{j}$ associated with the $j$-th residue contains primary sequence information and evolutionary information, and direct structural information derived from PDB templates:

$$
i_{j}=\left(i_{j}^{(E)}, i_{j}^{(T)}\right)
$$

where, assuming that $e$ units are devoted to sequence and

\begin{tabular}{|c|c|c|c|}
\hline $\max I D$ & baseline & PaleAle_H & PaleAle \\
\hline $95 \%$ & $61.1 \%$ & $64.8 \%$ & $53.8 \%$ \\
\hline $30 \%$ & $49.2 \%$ & $57.1 \%$ & $54.3 \%$ \\
\hline $20 \%$ & $45.6 \%$ & $55.5 \%$ & $54.4 \%$ \\
\hline
\end{tabular}
evolutionary information, and $t$ to structural information:

Table 2: Performances of the template-based 4-class solvent accessibility predictor (PaleAle_H) compared with a baseline predictor which copies the solvent accessibility of the best template (baseline) and with the ab initio solvent accessibility predictor (PaleAle).

Templates up to $95 \%, 30 \%$ and $20 \%$ maximum similarity allowed (maxID) for baseline and PaleAle_H. Performances measured only on residues for which a template has been identified. Results in 5-fold cross-validation on the S2I7I set (see text for details). 
Table 3: Performances of the two-class PaleAle and PaleAle_H compared with a number of recent methods on the Manesh dataset [39].

\begin{tabular}{cc}
\hline Method & \\
\hline PaleAle_H & $86.0 \%$ \\
PaleAle & $79.2 \%$ \\
NETASA [15] & $70.3 \%$ \\
[2I] & $78.1 \%$ \\
PP [47] & $78.1 \%$ \\
PredAcc [1I] & $70.7 \%$ \\
JNET [13] & $75.0 \%$ \\
ACCPro [16] & $77.2 \%$ \\
SABLE [17] & $77.6 \%$ \\
\hline
\end{tabular}

Performances of the various methods from [2I]. The class threshold is $25 \%$ for all methods. Templates up to $95 \%$ adopted by PaleAle_H.

$$
i_{j}^{(E)}=\left(i_{j, 1}^{(E)}, \ldots, i_{j, e}^{(E)}\right)
$$

and:

$$
i_{j}^{(T)}=\left(i_{j, 1}^{(T)}, i_{j, t}^{(T)}\right)
$$

Hence $i_{j}$ contains a total of $e+t$ components.

As in [19] $=$ = 25: beside the 20 standard amino acids, $\mathrm{B}$ (aspartic acid or asparagine), U (selenocysteine), X (unknown), Z (glutamic acid or glutamine) and · (gap) are considered. The input presented to the networks is the frequency of each of the 24 non-gap symbols, plus the overall frequency of gaps in each column of the alignment. I.e., if $n_{j k}$ is the total number of occurrences of symbol $j$ in column $k$, and $g_{k}$ the number of gaps in the same column, the $j^{\text {th }}$ input to the networks in position $k$ is:

$$
\frac{n_{j k}}{\sum_{v=1}^{24} n_{v k}}
$$

for $j=1 \ldots 24$, while the $25^{\text {th }}$ input is:

$$
\frac{g_{k}}{g_{k}+\sum_{v=1}^{24} n_{v k}}
$$

This input coding scheme is richer than simple 20-letter schemes and has proven effective in [19].

In the case of secondary structure prediction we use $t=10$ for representing structural information from the templates. Hence the total number of inputs for a given residue is $e+t=35$. The first 8 structural input units contain the average 8-class (DSSP style) secondary structure composition in the PDB templates, while the last 2 encode the average quality of the template column. Assume that $s_{p, j}$ is an 8-component vector encoding the DSSP-assigned
Table 4: Percentage of residues on which Porter_H and PaleAle_H disagree with the template-based part of their input (profile of secondary structure/solvent accessibility frequency from templates, in which each template is weighed by its cubed sequence similarity to the query - see Methods section for details).

\begin{tabular}{ccc}
\hline & Porter_H & PaleAle_H \\
\hline $\mathrm{e}<0.01$ & $8.6 \%$ & $14.4 \%$ \\
$\mathrm{e}>0.01$ & $30.8 \%$ & $21.6 \%$ \\
All & $9.9 \%$ & $21.2 \%$
\end{tabular}

Results on all residues for which a template exists (All), residues for which a template exists with BLAST e-value smaller than 0.01 ( $e<$ 0.01 ) and all residues for which a template exists after having removed templates with BLAST e-value smaller than 0.01 ( $e>0.01$ ). Results in 5 -fold cross-validation on the S217I set (see text for details).

8 -class secondary structure of $j$-th residue in the $p$-th template as follows:

$$
\begin{aligned}
& H=(1,0,0,0,0,0,0,0) \\
& G=(0,1,0,0,0,0,0,0) \\
& I=(0,0,1,0,0,0,0,0) \\
& E=(0,0,0,1,0,0,0,0) \\
& B=(0,0,0,0,1,0,0,0) \\
& S=(0,0,0,0,0,1,0,0) \\
& T=(0,0,0,0,0,0,1,0) \\
& \cdot=(0,0,0,0,0,0,0,1)
\end{aligned}
$$

Then, if $P$ is the total number of templates for a protein:

$$
\left(i_{j, 1}^{(T)}, \ldots, i_{j, 8}^{(T)}\right)=\frac{\sum_{p=1}^{P} w_{p} s_{p, j}}{\sum_{p=1}^{P} w_{p}}
$$

Where $w_{p}$ is the weight attributed to the $p$-th template. If the identity between template $p$ and the query is idp and the quality of a template (measured as X-ray resolution + R-factor/20, as in [33] - the lower the better) is $q_{s^{\prime}}$ then it is:

$$
w_{p}=i d_{p}^{3} / q_{p}
$$

Taking the cube of the identity between template and query drastically reduces the contribution of low-similarity templates when good templates are available. For instance a $90 \%$ identity template is weighed two orders of magnitude more than a $20 \%$ one. In preliminary tests 


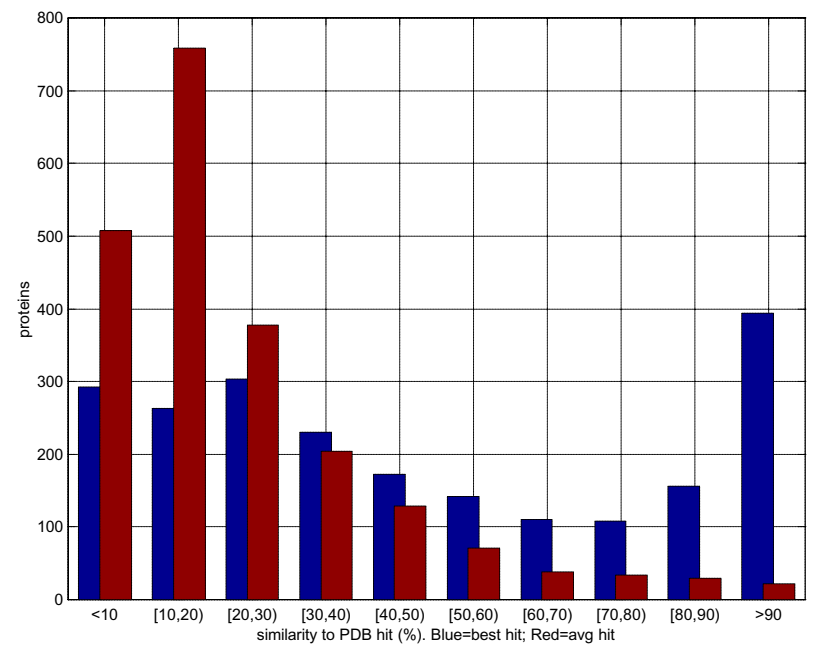

Figure 7

Distribution of best-hit (blue) and average (red) sequence similarity in the PSI-BLAST templates for the S2 171 set. Hits above $95 \%$ sequence similarity excluded.

(not shown) this measure performed better than a number of alternatives.

The final two units of $i_{j}$ encode the weighted average coverage and similarity of a column of the template profile as follows:

$$
i_{j, 9}^{(T)}=\frac{\sum_{p=1}^{P} w_{p} c_{p}}{\sum_{p=1}^{P} w_{p}}
$$

where $c_{p}$ is the coverage of the sequence by template $p$ (i.e. the fraction of non-gaps in the alignment), and

$$
i_{j, 10}^{(T)}=\frac{\sum_{p=1}^{P} w_{p} i d_{p}}{\sum_{p=1}^{P} w_{p}}
$$

It is worth noting how both structural information from templates and the two indices of template quality above are residue-based. For this reason, the case in which only templates covering fragments of a protein exist does not pose a problem for the method - the residues not covered by templates will simply have the section of the input with template information blank, and predictions will be based only on the sequence (and on sequence and template information transmitted by the forward and backwards memory chains). Template information for solvent accessibility is encoded similarly to secondary structure, except that 4 units are adopted to represent average sol- vent accessibility from PDB-derived templates (4 approximately equal classes). The two units encoding the profile quality are the same as in the secondary structure case. For the comparative experiments without templates, exactly same architectures are adopted, except that the part of the inputs $i_{j}^{(T)}$ representing the template profile is set to zero.

\section{Filtering BRNN}

We adopt a second filtering BRNN, similarly to [19]. The network is trained to predict secondary structures (or solvent accessibilities) given first-layer secondary structure (resp. accessibility) predictions. The $i$-th input to this second network includes the first-layer predictions in position $i$ augmented by first stage predictions averaged over multiple contiguous windows. That is, if $c_{j 1}, \ldots c_{j m}$ are the outputs in position $j$ of the first stage network corresponding to estimated probabilities of secondary structure or solvent accessibility $j$ being in class $m$, the input to the second stage network in position $j$ is the array $I_{j}$ :

$$
\begin{array}{r}
\sum_{h=k_{-p}-w}^{I_{j}}=\left(c_{j 1}, \ldots, c_{j m},\right. \\
\sum_{h=k_{p}-w}^{k_{-p}+w} c_{h 1}, \ldots, \sum_{h=k_{-p}-w}^{k_{-p}+w} c_{h m}, \\
k_{p}+w \\
\left.\sum_{h=k_{p}-w} c_{h m}\right)
\end{array}
$$

where $k_{f}=j+f(2 w+1), 2 w+1$ is the size of the window over which first-stage predictions are averaged and $2 p+1$ is the number of windows considered. In the tests we use $w=7$ and $p=7$, as in [19]. This means that 15 contiguous, non-overlapping windows of 15 residues each are considered, i.e. first-stage outputs between position $j-112$ and $j$ +112 , for a total of 225 contiguous residues, are taken into account to generate the input to the filtering network in position $j$. This input contains a total of $16 \mathrm{~m}$ real numbers: $m$ representing the $m$-class output of the first stage in position $j ; 15 m$ representing the $m$-class outputs of the first-stage averaged over each of the 15 windows. $m$ is 3 in the case of secondary structure prediction and 4 for (4class) solvent accessibility prediction.

\section{Training, Ensembling}

Five two-stage BRNN models are trained independently and ensemble averaged to build the final predictor. Differences among models are introduced by two factors: stochastic elements in the training protocol, such as different initial weights of the networks and different shuffling of the examples; different architecture and number of free parameters of the models. The training strategy is identical to that adopted for Porter [19]: 1000 epochs of training 
are performed for each model; the learning rate is halved every time we do not observe a reduction of the error for more than 50 epochs. The size and architecture of the models, apart from differences caused by the different number of inputs, is the same as Porter's. The number of free parameter per model ranges between 5,800 and 8,000 . The template-based models are only slightly larger (on average $7 \%$ more free parameters) than the corresponding $a b$ initio ones. Averaging the 5 models' outputs leads to classification performance improvements between $1 \%$ and $1.5 \%$ over single models. Furthermore a copy of each of the 5 models is saved at regular intervals (100 epochs) during training. Stochastic elements in the training protocol (similar to that described in [14]) guarantee that differences during training are non-trivial. An ensemble of a total of 45 such models yields a further slight improvement over the ensemble of 5 models.

\section{Authors' contributions}

GP suggested the protocol for incorporating templates, designed the overall pipeline, and implemented and tested the secondary structure prediction stage and the web version of the servers. AJMM designed and implemented the stage for homology detection. CM implemented and tested the predictor of solvent accessibility and analysed most of the results. AV contributed to the implementation of all stages and to the analysis of the results. The manuscript was written by GP, AJMM and AV and approved by all authors.

\section{Acknowledgements}

This work is supported by Science Foundation Ireland grants 04/BR/ CS0353 and 05/RFP/CMS0029, grant RP/2005/219 from the Health

Research Board of Ireland, a UCD President's Award 2004, and an Embark Fellowship from the Irish Research Council for Science, Engineering and Technology to AV.

\section{References}

I. [Http://distill.ucd.ie/].

2. Bradley P, Chivian D, Meiler J, Misura K, Rohl C, Schief W, Wedemeyer W, Schueler-Furman O, Murphy P, Schonbrun J, Strauss C, Baker D: Rosetta predictions in CASP5: Successes, failures, and prospects for complete automation. Proteins 2003, 53(S6):457-468.

3. Jones D: GenTHREADER: an efficient and reliable protein fold recognition method for genomic sequences. I Mol Biol 1999, 287:797-8I5

4. Karchin R, Cline M, Mandel-Gutfreund Y, Karplus K: Hidden markov models that use predicted local structure for fold recognition: alphabets of backbone geometry. Proteins 2003, 5 I (4):504-I4.

5. Przybylski D, Rost B: Improving Fold Recognition Without Folds. Journal of Molecular Biology 2004, 341:255-269.

6. Rost B, Yachdav G, Liu J: The PredictProtein server. Nucleic Acids Research 2004, 32:W321-326.

7. Salamov A, Solovyev V: Prediction of protein secondary structure by combining nearest-neighbor algorithms and multiple sequence alignments. Journal of Molecular Biology 1995, 247: I I-5.

8. Rost B: PHD: predicting ID proteins structure by profile based neural networks. Meth in Enzym 1996, 266:525-539.

9. Jones D: Protein secondary structure prediction based on position-specific scoring matrices. J Mol Biol 1999, 292:195-202.
10. Baldi P, Brunak S, Frasconi P, Soda G, Pollastri G: Exploiting the past and the future in protein secondary structure prediction. Bioinformatics 1999, 15:937-946.

II. Mucchielli-Giorgi M, Hazout S, Tuffery P: PredAcc: prediction of solvent accessibility. Bioinformatics 1999, I5(2): I76-7.

12. Petersen T, Lundegaard C, Nielsen M, Bohr H, Bohr J, Brunak S, Gippert G, Lund O: Prediction of protein secondary structure at $80 \%$ accuracy. Proteins 2000, 41 (1): $17-20$.

13. Cuff J, Barton G: Application of multiple sequence alignment profiles to improve protein secondary structure prediction. Proteins 2000, 40(3):502-II.

14. Pollastri G, Przybylski D, Rost B, Baldi P: Improving the prediction of protein secondary structure in three and eight classes using recurrent neural networks and profiles. Proteins 2002, 47:228-235.

15. Ahmad S, Gromiha M: NETASA: neural network based prediction of solvent accessibility. Bioinformatics 2002, I 8(6):8|9-24.

16. Pollastri G, Fariselli P, Casadio R, Baldi P: Prediction of coordination number and relative solvent accessibility in proteins. Proteins 2002, 47: 142-235.

17. Adamczak R, Porollo A, Meller J: Accurate prediction of solvent accessibility using neural networks-based regression. Proteins 2004, 56(4):753-67.

18. Wagner M, Adamczak R, Porollo A, Meller J: Linear regression models for solvent accessibility prediction in proteins. Journal of Computational Biology 2005, I 2(3):355-69.

19. Pollastri G, McLysaght A: Porter: a new, accurate server for protein secondary structure prediction. Bioinformatics 2005, 2I(8): $17 \mid 9-20$.

20. Qin S, Pan X: Predicting Protein Secondary Structure and Solvent Accessibility with and Improved Multiple Linear Regression Method. Proteins 2005, 61:473-80.

21. Nguyen M, Rajapakse J: Prediction of Protein Relative Solvent Accessibility With a Two-Stage SVM Approach. Proteins 2005, 59:30-7.

22. Montgomerie S, Sundaraj S, Gallin W, Wishart D: Improving the Accuracy of Protein Secondary Structure Prediction Using Structural Alignment. BMC Bioinformatics 2006, 7:30I.

23. Altschul S, Madden T, Schaffer A: Gapped blast and psi-blast: a new generation of protein database search programs. Nucl Acids Res 1997, 25:3389-3402.

24. Berman H, Westbrook J, Feng Z, Gilliland G, Bhat T, Weissig H, Shindyalov I, Bourne P: The Protein Data Bank. Nucl Acids Res 2000, 28:235-242 [http://pdbbeta.rcsb.org/pdb/Welcome.do].

25. Orengo C, Bray J, Hubbard T, Lo Conte L, Sillitoe I: Analysis and assessment of ab initio three-dimensional prediction, secondary structure, and contacts prediction. Proteins: Structure, Function and Genetics 1999, 37(S3): I49-170.

26. Lesk A, Lo Conte L, Hubbard T: Assessment of novel fold targets in CASP 4: predictions of three-dimensional structures, secondary structures, function and genetics. Proteins: Structure, Function and Genetics 200I, S5:98-II8.

27. Moult J, Fidelis $\mathrm{K}$, Zemla A, Hubbard $\mathrm{T}$ : Critical assessment of methods of protein structure prediction (CASP)-round $\mathrm{V}$. Proteins 2003, 53(Suppl 6):334-339.

28. Moult J, Fidelis K, Tramontano A, Rost B, Hubbard T: Critical Assessment of Methods of Protein Structure Prediction (CASP)-Round VI. Proteins 2005, 6 I (Suppl 6):3-7.

29. Rost $B$, Sander C: Prediction of protein secondary structure at better than 70\% accuracy. J Mol Biol 1993, 232:584-599.

30. Cuff JA, Barton GJ: Application of multiple sequence alignments profiles to improve protein secondary structure prediction. Proteins: Structure, Function and Genetics 2000, 40(3):502-5II.

31. Eyrich V, Marti-Renom M, Przybylski D, Madhusudan M, Fiser A, Pazos F, Valencia A, Sali A, Rost B: EVA: continuous automatic evaluation od protein structure prediction servers. Bioinformatics 200I, 17:|242-I25I.

32. Cheng J, Baldi P: A machine learning information retrieval approach to protein fold recognition. Bioinformatics 2006, 22(I 2): | 456-63.

33. Hobohm U, Sander C: Enlarged representative set of protein structures. Protein Sci 1994, 3:522-24 [http://bioinfo.tg.fh-gies sen.de/pdbselect/]. 
34. Kabsch W, Sander C: Dictionary of protein secondary structure: pattern recognition of hydrogen-bonded and geometrical features. Biopolymers 1983, 22:2577-2637.

35. Frishman D, Argos P: Knowledge-based protein secondary structure assignment. Proteins 1995, 23(4):566-579.

36. Fourrier L, Benros C, de Brevern A: Use of a structural alphabet for analysis of short loops connecting repetitive structures. BMC Bioinformatics 2004, 5:58.

37. Ceroni A, Frasconi P, Pollastri G: Learning Protein Secondary Structure from Sequential and Relational Data. Neural Networks 2005, I 8(8): 1029-39.

38. Sim J, Kim S, Lee J: Prediction of protein solvent accessibility using fuzzy k-nearest neighbor method. Bioinformatics 2005, 2 I ( I 2):2844-9.

39. Naderi-Manesh H, Sadeghi M, Araf S, Movahedi A: Prediction of protein surface accessibility with information theory. Proteins 200I, 42(4):452-9.

40. Riis SK, Krogh A: Improving prediction of protein secondary structure using structured neural networks and multiple sequence alignments. J Comp Biol 1996, 3(I): I63-183.

4I. Baldi P, Pollastri G, Andersen CAF, Brunak S: Matching protein $\beta$ sheet partners by feedforward and recurrent neural networks. In Proceedings of the 2000 Conference on Intelligent Systems for Molecular Biology (ISMB00), La Jolla, CA Volume 8. Menlo Park, CA: AAAI Press; 2000:25-36.

42. Cheng J, Baldi P: Three-stage prediction of protein $\beta$-sheets by neural networks, alignments and graph algorithms. Bioinformatics 2005, 2 I :i75-i84.

43. Pollastri G, Baldi P: Prediction of Contact Maps by Recurrent Neural Network Architectures and Hidden Context Propagation from All Four Cardinal Corners. Bioinformatics 2002 I 8(SuppI I):S62-S70.

44. Baldi P, Pollastri G: The Principled Design of Large-Scale Recursive Neural Network Architectures - DAG-RNNs and the Protein Structure Prediction Problem. Journal of Machine Learning Research 2003, 4(Sep):575-602.

45. Vullo A, Walsh I, Pollastri G: A two-stage approach for improved prediction of residue contact maps. BMC Bioinformatics 2006, 7:180.

46. Krieger E, Hooft R, Nabuurs S, Vriend G: PDBFinderll - a database for protein structure analysis and prediction. 2004 in press. http://swift.cmbi.ru.nl/gv/pdbfinder/

47. Gianese G, Bossa F, Pascarella S: Improvement in prediction of solvent accessibility by probability profiles. Protein Engineering 2003, I 6( I 2):987-92.
Publish with Biomed Central and every scientist can read your work free of charge

"BioMed Central will be the most significant development for disseminating the results of biomedical research in our lifetime. "

Sir Paul Nurse, Cancer Research UK

Your research papers will be:

- available free of charge to the entire biomedical community

- peer reviewed and published immediately upon acceptance

- cited in PubMed and archived on PubMed Central

- yours - you keep the copyright
BioMedcentral 